Gut, 1973, 14, 83-88

\title{
Cholesterol and bile salt studies on the bile of patients with cholesterol gallstones
}

\author{
F. HELLER AND IAN A. D. BOUCHIER ${ }^{1}$ \\ From the Department of Medicine, The Royal Free Hospital, London
}

SUMMARY Gallbladder bile from patients with cholesterol stones and control patients without cholesterol stones was analysed for the relative concentrations of bile salts, phospholipids, and cholesterol. It was not possible to distinguish stone-forming from control biles when these were plotted on triangular coordinates. There was a significant increase in the dihydroxy:trihydroxy bile salt ratio in the patients with cholesterol gallstones. The maximum cholesterol holding capacity for the bile salts was determined and no difference could be detected in the ability of the bile salts from either cholesterol stone-containing and control bile samples to dissolve cholesterol.

It is generally agreed that the pathogenesis of cholesterol gallstones is related to the capacity of the bile to maintain cholesterol in a mixed micellar solution. On the basis of experiments in vitro carried out at $25^{\circ} \mathrm{C}$ Bourges, Small, and Dervichian (1967) constructed a phase diagram with four coordinates: bile salt, lecithin, cholesterol, and water. This permitted a prediction of the physical state of an aqueous system containing known concentrations of cholesterol, lecithin, and bile salts. This work was extended to human bile samples and it was demonstrated that when gallbladder bile having a total solid concentration of 5 to $20 \%$ by weight was plotted on triangular coordinates the bile from patients with cholesterol gallstones fell in the region near or outside the line of maximal cholesterol solubility whereas bile samples without gallstones fell within the micellar phase. Thus the cholesterol in bile-containing cholesterol stones can be conceived of existing in at least two phases: a micellar phase with microcrystals and/or liquid crystals (Admirand and Small, 1968).

Recent studies on the bile physico-chemistry have failed to confirm the clear distinction between normal and cholesterol stone-containing biles (Nakayama and van der Linden, 1970; Dam, 1971; Holzbach and Marsh, 1971). The present investigation was undertaken in an attempt to determine whether it is possible to distinguish gallstone-containing bile from

${ }^{1}$ Please address requests for reprints to: Ian A. D. Bouchier, MD, FRCP, Reader in Medicine, Department of Medicine, The Royal Free Hospital, Gray's Inn Road, London WC1X 8LF

Received for publication 5 December 1972. bile without gallstones. Bile samples were plotted on triangular coordinates and related to the line of maximum micellar solubility as described by Admirand and Small (1968). A comparison was made of the dihydroxy : trihydroxy bile salt ratio in the bile samples; finally an attempt was made to evaluate the capacity of the bile salts to hold cholesterol in solution by calculating the 'maximum cholesterol-holding capacity' of the samples. It was concluded that it was not possible to make a clear distinction between normal and cholesterol gallstonecontaining bile either on the basis of the triangular coordinate plot or from the capacity in vitro of the bile salts to dissolve cholesterol.

\section{Materials and Methods}

Gallbladder bile samples were obtained from 28 patients by needle aspiration of the gallbladder. Twenty-five samples were obtained at the time of abdominal surgery. In three patients bile was obtained at necropsy undertaken within 18 hours of death (Biss, Mikkelson, Lewis, and Taylor, 1971). The cystic duct was clamped to avoid contamination with hepatic bile and the gallbladders were aspirated completely thereby avoiding possible sampling errors due to stratification (Tera, 1960). None of the samples was grossly contaminated with blood. The samples were divided into two groups. Biles from gallbladders without stones (9) or containing pigment stones (3) were regarded as controls. Control subjects had no clinical or radiological evidence of gallbladder disease; the patients had undergone 
surgery on the upper gastrointestinal tract and the biliary tree was shown to be normal at the time of operation. The three patients with pigment stones had normally functioning gallbladders on cholecystography. There were 16 samples from gallbladders shown to contain cholesterol stones and these were regarded as abnormal. These patients had functioning gallbladders on oral cholecystography.

The bile samples were either frozen at $-10^{\circ} \mathrm{C}$ or extracted within half an hour of collection. Frozen samples were thawed and all samples were well shaken before analysis. They were not subjected to centrifugation or ultrafiltration. All samples were checked by thin-layer chromatography for the presence of free bile acids and none was detected.

The bile was extracted in a mixture of $50 \%$

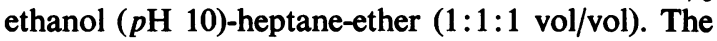
bile salts were isolated by evaporation of the ethanolic phase, dissolved in $50 \%$ methanol ( $p \mathrm{H} 10)$, and estimated by the hydroxysteroid dehydrogenase method of Iwata and Yamasaki (1964). The bile acids were separated by silica gel thin-layer chromatography (TLC) using the system of ethylene dichloride-acetic acid-water $(1: 1: 0 \cdot 1 \mathrm{vol} / \mathrm{vol})$ of Gregg (1966). This system separates bile acid mixtures into their free and taurine- and glycine-conjugated fractions and within these groups into trihydroxy, dihydroxy, and monohydroxy bile acids but does not separate either the conjugated or free forms of deoxycholic acid from those of chenodeoxycholic acid. The bile acid fractions were located with iodine and eluted from the silica gel with $50 \%$ ethanol. After evaporating off the different fractions the residues were dissolved in methanolic pyrophosphate buffer and the bile acids measured by the enzymatic method. The recovery of known amounts of cholic, chenodeoxycholic, and deoxycholic acids carried through the chromatographic procedure ranged from 64 to $100 \%$ with a mean of $85 \%$. Cholesterol was extracted from the upper heptane-ether phase and assayed by the method of Watson (1960). Phospholipids were measured by the method of King (1932) after Folch extraction of the bile (Folch, Lees, and Stanley, 1957).

DETERMINATION OF THE MAXIMUM CHOLESTEROL-HOLDING CAPACITY

One hundred $\mu \mathrm{mol}$ of bile acid was isolated from the bile of each patient by extracting a calculated volume of bile with $50 \%$ ethanol $(p \mathrm{H} \mathrm{10)}$. The recovery of known amounts of bile acid added to the bile ranged from 97.4 to $101.0 \%$. The ethanolic solution was introduced into a conical flask and taken to dryness. Purified egg lecithin (BDH Chemicals Ltd, Poole, England), shown to be more than $95 \%$ pure by TLC (Skipski, Peterson, and
Barclay, 1964), was dissolved in a methanolchloroform solution. A volume of this solution containing $50 \mu \mathrm{mol}$ lecithin was added to the flask containing the bile acids. To this was added 10-15 $\mathrm{mg}$ cholesterol dissolved in pure chloroform to ensure that there was an excess of chloroform in the mixture. The solvents were evaporated by gentle heating under a nitrogen stream. The dry residue was mixed with $1 \mathrm{ml}$ phosphate buffer $(p \mathrm{H} \mathrm{7.37)}$ and the flask sealed in a nitrogen atmosphere. The mixture was incubated at $37^{\circ} \mathrm{C}$ in a shaking water bath for two days and thereafter left standing at $37^{\circ}$ for a further 24 hours. At the end of the three-day period the mixture was completely aspirated into a syringe and immediately transferred to a millipore filter (pore size $0.22 \mu$ ). Filtration was performed at $37^{\circ} \mathrm{C}$ and the clear filtrate estimated for cholesterol content. The maximum cholesterol-holding capacity of the bile salts was expressed as $\mu \mathrm{mol}$ cholesterol per $\mathrm{ml}$ of clear filtrate. All experiments were performed in duplicate.

Because the concentrations of bile acids and lecithin were identical in each experiment the maximum cholesterol-holding capacity depended upon the composition of the bile acids extracted from the original bile samples. Thus the experiments permitted a comparison to be made of the capacity of bile salts from control and cholesterol gallstonecontaining biles to dissolve cholesterol. This method is valid only if cholesterol is held in clear solution during the filtration procedure. However, we did observe that all the filtrates became unstable unless they were very carefully handled. For example, the solution readily became cloudy on cooling to room temperature or agitation and this physical state could not be reversed on heating.

\section{Results}

QUANTITATIVE ANALYSIS OF THE BILE

The mean values for the biliary lipids from patients without and with cholesterol gallstones are shown in the Table. The concentrations of the bile salts, phospholipids, and cholesterol were not significantly different between the two groups. The ratio of bile salts + phospholipids:cholesterol (Isaksson, 1954) was 18.7 \pm 12.6 in control patients and this was not significantly different from patients with cholesterol gallstones $(13 \cdot 3 \pm 8 \cdot 1)$. When the results were plotted as single points on a triangular coordinate diagram two of 12 control samples fell outside the micellar zone while 10 of 16 samples of cholesterol gallstonecontaining bile fell within the micellar zones (Fig. 1). Taking the group as a wholc $20(71.5 \%)$ of the samples were plotted to lie inside the micellar zone. It is clear that there was considerable overlap between 


\begin{tabular}{|c|c|c|c|c|c|c|}
\hline & Bile Salts & $\begin{array}{l}\text { Phospholipid } \\
\text { (mmol/l) }\end{array}$ & Cholesterol & Bile Salts & $\begin{array}{l}\text { Phospholipid } \\
(\% \text { total } \mathrm{mmol})\end{array}$ & Cholesterol \\
\hline $\begin{array}{l}\text { Control } \\
\text { Mean } \\
\pm 1 \text { SD }\end{array}$ & $\begin{array}{r}145.9 \\
56.9\end{array}$ & $\begin{array}{l}33 \cdot 9 \\
12 \cdot 3\end{array}$ & $\begin{array}{r}13 \cdot 0 \\
8 \cdot 5\end{array}$ & $\begin{array}{r}75 \cdot 3 \\
5 \cdot 5\end{array}$ & $\begin{array}{r}17 \cdot 6 \\
2 \cdot 7\end{array}$ & $\begin{array}{l}6.9 \\
3.4\end{array}$ \\
\hline $\begin{array}{l}\text { Cholesterol Stone } \\
\text { Mean } \\
\pm 1 \text { SD }\end{array}$ & $\begin{array}{r}137 \cdot 2 \\
65 \cdot 4\end{array}$ & $\begin{array}{l}33 \cdot 3 \\
18 \cdot 0\end{array}$ & $\begin{array}{r}14 \cdot 6 \\
7 \cdot 3\end{array}$ & $\begin{array}{r}73 \cdot 7 \\
7 \cdot 7\end{array}$ & $\begin{array}{r}17 \cdot 6 \\
5.9\end{array}$ & $\begin{array}{l}8 \cdot 6 \\
3 \cdot 8\end{array}$ \\
\hline
\end{tabular}

Table Gallbladder bile composition from 12 control subjects and 16 patients with cholesterol stones

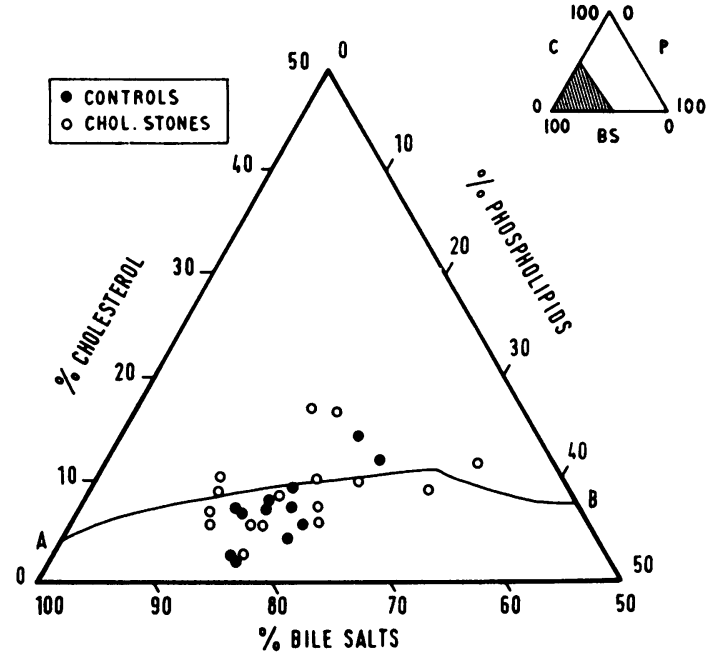

Fig. 1 A comparison of gallbladder bile obtained from contrcl subjects and patients with cholesterol gallstones. Mean mmol \% bile salts, phospholipids, and cholesterol are plotted on triangular coordinates. The graph shows details of the lower left hand corner of the entire plot (insert). The line $A B$ of maximal cholesterol solubility is taken from the model system described by Admirand and Small (1968).

the two groups (Fig. 2) and that the line of maximum micellar solubility for cholesterol as described by Admirand and Small (1968) did not, in our experience, distinguish cholesterol stone-containing bile from bile without such stones.

\section{QUALITATIVE ANALYSIS OF THE BILE SALTS}

The values of the dihydroxy:trihydroxy bile salt ratios are shown in Figure 3. The mean ratio in the cholesterol gallstone group was $2.74 \pm 1.42( \pm 1$ $\mathrm{SD})$ which was significantly greater $(\mathrm{P}<0.025$, Student's $t$ test) than the ratio in control bile samples $(1.68 \pm 0.57)$.

\section{DETERMINATION OF THE MAXIMUM} CHOLESTEROL-HOLDING CAPACITY Similar amounts of cholesterol were held in solution

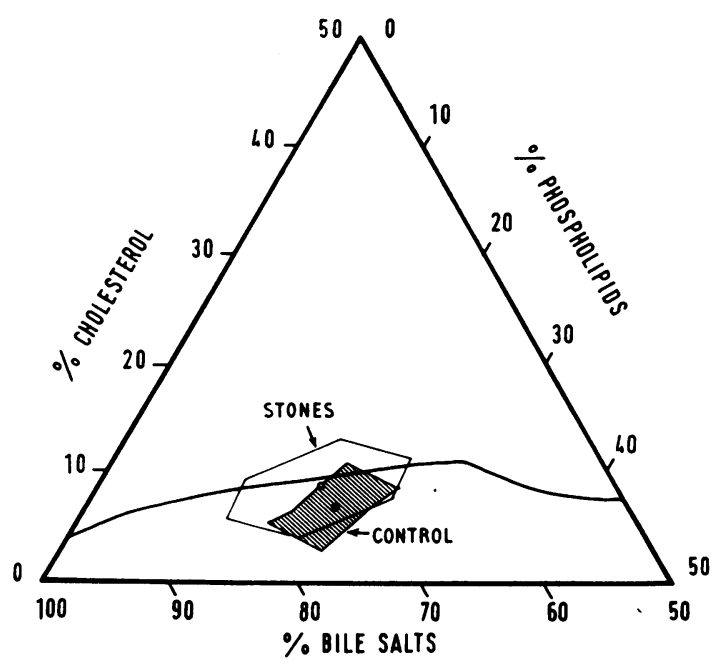

Fig. 2 Mean $\pm S D$ of control (shaded area) and cholesterol stone-containing (unshaded area) bile plotted on triangular coordinates.

by the bile salts of control and cholesterol stonecontaining biles under the conditions of the experiment despite the difference in the composition of the bile salts. The mean maximum cholesterol-holding capacity for control samples was $21 \cdot 0 \pm 2 \cdot 6( \pm 1$ SD) $\mu \mathrm{mol}$ cholesterol $/ \mathrm{ml}$ filtrate and $20.0 \pm 3.6$ $\mu \mathrm{mol}$ cholesterol $/ \mathrm{ml}$ filtrate for cholesterol stonecontaining biles (Fig. 4). There was no relationship between the maximum cholesterol-holding capacity and the dihydroxy:trihydroxy bile salt ratio.

\section{Discussion}

This study has failed to confirm the earlier report of Admirand and Small (1968) that a clear distinction can be made between cholesterol stone-containing bile and stone-free samples using a triangular coordinate plot. We found that there was considerable overlap between the two groups and that $16.6 \%$ of the control samples were outside the micellar zone whereas $62.5 \%$ of the samples from gallbladders 


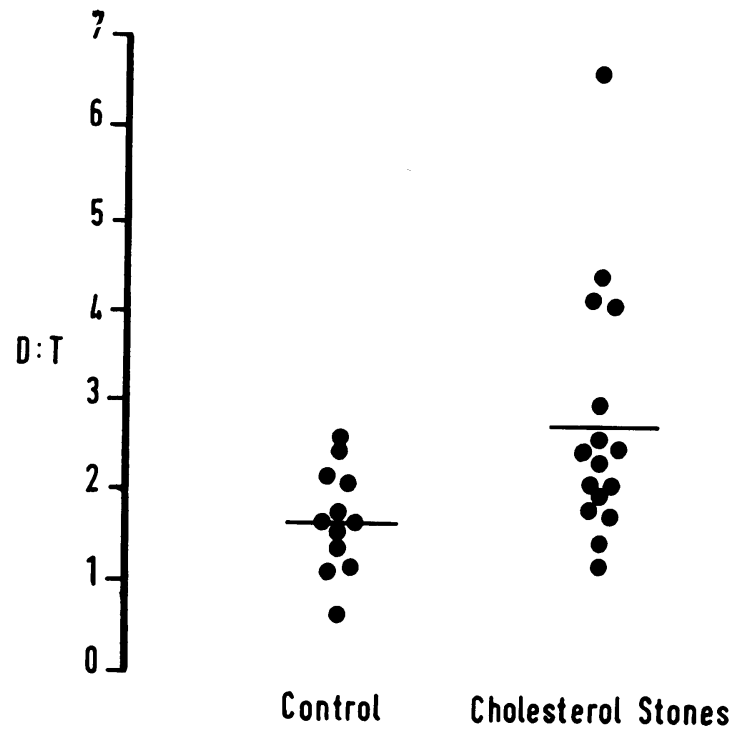

Fig. 3 The ratio of dihydroxy:trihydroxy bile salts in bile of control patients and patients with cholesterol stones. Mean values indicated by solid line.

with stones fell within the micellar zone. This observation confirms the report of Nakayama and van der Linden (1970) who failed to show a clear difference between bile samples with and without gallstones. Approximately $20 \%$ of their patients with gallstones had bile in a single micellar phase whereas $75 \%$ of bile samples without stones fell outside the micellar phase. Similar findings were reported by Holzbach and Marsh (1971). Using different criteria for assessing cholesterol solubility, Dam, Kruse, Prange, Kallehauge, Fenger, and Krogh Jensen (1971) were unable to separate bile from patients with gallstones from those with peptic ulcer. Dam (1971) believed that the majority of samples in their study would have fallen in the micellar zone if plotted on triangular coordinates. Smallwood, Jablonski, McLelland, and Watts (1972) reported that $40 \%$ of gallbladder bile samples and $35 \%$ of hepatic bile samples from gallstone patients fell within the micellar zone although none of their control samples fell above the line of maximum cholesterol micellar solubility. In an analysis of bile obtained by duodenal drainage Danzinger, Hofmann, Schoenfield, and Thistle (1972) found that six of seven patients with gallstones had bile within the micellar zone; using a similar method for obtaining bile, Dowling, Bell, and White (1972) observed that nearly $25 \%$ of control subjects had bile plotted to fall outside the micellar zone.

There are a number of theoretical reasons why it

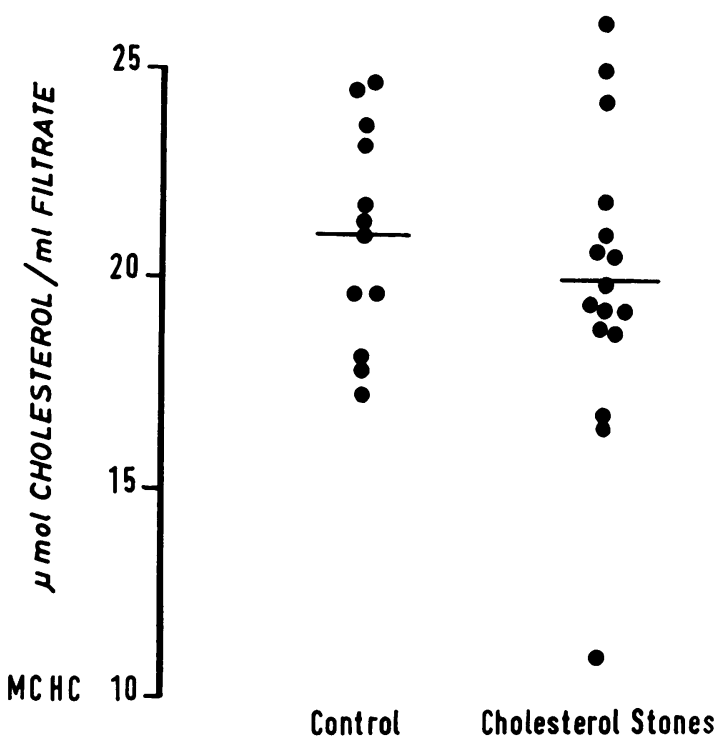

Fig. 4 Maximum cholesterol-holding capacity of bile salts from control patients with cholesterol stones. Mean values indicated by solid line.

may not be possible at present to distinguish physicochemical differences between normal biles and biles with cholesterol stones: (1) the normal bile may be in a 'lithogenic' phase; (2) the line of maximum micellar solubility for cholesterol as derived by Admirand and Small may not be correct for human bile; (3) the methods for handling bile samples may introduce errors; (4) there may be differences in the cholesterol-dissolving capacity of different bile salts or lecithin; and (5) the composition of the bile may not be constant.

We believe that the concept of a clearly definable 'lithogenic' bile which predicts gallstone disease (Nakayama and van der Linden, 1970; Vlahcevic, Bell, and Swell, 1970; Thistle and Schoenfield, 1971) may have been introduced prematurely. Thus far there is only limited evidence to show that subjects with a bile composition lying outside the micellar zone have subsequently formed gallstones. Furthermore the argument is based on the concept that all patients with gallstones have bile which falls outside the micellar zone, ie, that bile with such a cholesterol: bile salts and lecithin ratio is necessarily 'lithogenic'; however, it has now been shown that a considerable number of gallstone patients have bile within the micellar zone and which, under different circumstances, would be considered 'non-lithogenic' Certainly biles containing stones can be called 'lithogenic'. However the mechanism of gallstone formation is undoubtedly complex and a system of 
prediction based solely on the plotting of bile on triangular coordinates may not be sufficient to include all the factors necessary in predicting stone formation.

There have been recent attempts to redefine the maximum limits of cholesterol micellar solubility (Niederhiser and Roth, 1968; Hegardt and Dam, 1971; Holzbach and Marsh, 1972). The data suggest that the maximum cholesterol solubility may be less than previously described and therefore a larger number of bile samples would lie above the line. It may well be that the concept of a 'line' separating a one-phase from a two-phase system is erroneous and that it would be more correct to think of an 'area of instability' separating the two stable phases.

In our studies the bile samples were neither centrifuged nor filtered and all samples were well mixed before analysis. Therefore it appears unlikely that failure of discrimination is the result of a sampling error.

It has previously been shown that alterations in the composition of the biliary lecithins are unlikely to influence the cholesterol-dissolving capacity of bile (Saunders and Wells, 1969: Hegardt and Dam, 1971). There is disagreement regarding the dihydroxy: trihydroxy bile salt ratio in gallstone disease. The relevant literature has been summarized by van der Linden (1971) who suggested that a trend could be detected towards a greater dihydroxy : trihydroxy ratio in patients with gallstone disease. Our results are in keeping with van der Linden's observations, but in contrast to other authors we were able to show that the ratio was significantly greater in cholesterol stone-containing biles and this may be a reflection of the material included in this series. Invitro studies indicate that the dihydroxy bile salts dissolve more cholesterol than trihydroxy bile salts in the absence of lecithin (Yoshimuta, 1960; Niederhiser and Roth, 1968; Hegardt and Dam, 1971; Earnest and Admirand, 1971). In the presence of lecithin there is little or no difference in the cholesterol-solubilizing capacity of the different bile salts (Niederhiser and Roth, 1968; Hegardt and Dam, 1971). The present study indicated that differences in bile composition, particularly the dihydroxy: trihydroxy bile salt ratios, were not reflected in the capacity of the bile salts to dissolve cholesterol.

The reason for the increased quantity of dihydroxy bile salts in patients with gallstones has not been explained but might reflect altered liver synthesis in the presence of a reduced bile salt pool for it is known that increased hepatic bile salt synthesis may be accompanied by an increase in the dihydroxy chenodeoxycholic bile salt content of bile (Dowling, 1972). The gallbladder too might influence the bile salt pattern, because if it is relatively or completely non-functioning - an 'autocholecystectomy'-then the bile salt profile might resemble that of a patient with cholecystectomy where the dihydroxy deoxycholate bile salt forms an increased proportion of the bile salt pool (Pomare and Heaton, 1972).

At present the most attractive explanation for our failure to differentiate stone-containing bile from controls is that the composition of bile which is secreted by the liver may not be constant (Smallwood et al, 1972; Boyer, Maddrey, Bloomer, Tilson, and Wright, 1972). It is conceivable that the bile composition may fluctuate between a stable and a relatively unstable clear micellar solution. What factors might determine any variation in bile composition are unknown. Although it is apparent that it is not possible to distinguish stone-containing from normal gallbladder or hepatic bile on the basis of the analysis of random samples it is certain from all the data published that cholesterol stone-containing bile has a tendency to contain relatively more cholesterol. Our studies on the maximum cholesterolholding capacity indicate how unstable a clear solution with a high content of cholesterol can be. If carefully handled, at the temperature of the experiment $\left(37^{\circ} \mathrm{C}\right)$, the filtrate remained clear and cholesterol was in solution. This solution can be considered to be supersaturated (Small, 1968) but the cholesterol content was the same as in the mixtures studied by Admirand and Small (1968) which were plotted to lie on the line of maximum cholesterol solubility, and, according to the formula published by Niederhiser and Roth (1968), for cholesterol solubility in a mixture of bile salts and lecithin.

We propose that there is a zone on the phase diagram falling on either side of the line derived by Admirand and Small in which cholesterol is held in a clear but relatively unstable micellar phase. It may be that during some periods of the day this is the state in which cholesterol is normally secreted by the liver. Gallbladder bile will represent a mixture of stable and unstable hepatic bile and this in itself may not be abnormal. The presence of unstable bile is an important but not the sole factor in lithogenesis and the prediction of lithogenesis. In considering stone formation and the potential towards stone formation it is essential to take into account those factors in the gallbladder which permit nucleation, crystal formation, and stone growth.

We wish to thank Mrs P. Kyd for advice and assistance and Dr J. Sutor for analysis of the gallstones. We also wish to thank our surgical colleagues Mr I. Irving, FRCS, Mr W. W. Wilson, FRCS, Dr G. Therasse, and Dr G. Ninane for collecting operative samples of bile. Ian A. D. Bouchier is in support of a grant from the Medical Research Council. 


\section{References}

Admirand, W. H., and Small, D. M. (1968). The physicuchemical basis of cholesterol gallstone formation in man. J. clin. Invest., 47, 1043-1052.

Biss, K., Ho, K-J. Mikkelson, B., Lewis, L., and Taylor, C. B. (1971). Some unique biologic characteristics of the Masai of East Africa. New Engl. J. Med., 284, 694-699.

Bourges, M. Small, D. M., and Dervichian, D. G. (1967). Biophysics of lipidic associations. III. The quaternary systems lecithinbile salt-cholesterol-water. Biochim. biophys. Acta (Amst), 144, 189-201.

Boyer, J. L., Maddrey, W. C., Bloomer, J. R., Tilson, D., and Wright, H. (1972). Intermittent secretion of lithogenic bile by the liver of patients with previous gallstones. (Abstr.). Gastroenterology, $62,868$.

Dam, H. (1971). Determinants of cholesterol cholelithiasis in man and animals. Amer. J. Med., 51, 596-613.

Dam, H., Kruse, I., Prange, I., Kallehauge, H. E., Fenger, H. J., and Krogh Jensen, M. (1971). Studies on human bile. III. Composition of duodenal bile from healthy young volunteers compared with composition of bladder bile from surgical patients with and without uncomplicated gallstone disease. $Z$. Ernährungsw., 10, 160-177.

Danzinger, R. G., Hofmann, A. F., Schoenfield, L. J., and Thistle, J. L. (1972). Dissolution of cholesterol gallstones by chenodeoxycholic acid. New Engl. J. Med., 286, 1-8.

Dowling, R. H. (1972). The enterohepatic circulation. Gastroenterology, 62, 122-140.

Dowling, R. H., Bell, G. D., and White, J. (1972). Lithogenic bile in patients with ileal dysfunction. Gut, 13, 415-420.

Earnest, D. E., and Admirand, W. H. (1971). The effects of individual bile salts in cholesterol solubilization and gallstone dissolution. (Abstr.). Gastroenterology, 60, 772.

Folch, J., Lees, M., and Stanley, G. H. S. (1957). A simple method for the isolation and purification of total lipides from animal tissues. J. biol. Chem., 226, 497-509.

Gregg, J. A. (1966). New solvent systems for thin-layer chromatography of bile acids. J. Lipid Res., 7, 579-581.

Hegardt, F. G., and Dam, H. (1971). The solubility of cholesterol in aqueous solutions of bile salts and lecithin. Z. Ernährungsw., 10, 223-233.

Holzbach, R. T., and Marsh, M. (1971). Definition of cholesterol solubility in human bile. (Abstr.) Gastroenterology, 60, 677
Holzbach, R. T., and Marsh, M. (1972). A redefinition of maximum cholesterol solubility (MCS) in model solutions of bile salts and lecithin. (Abstr.). Gastroenterology, 62, 194.

Isaksson, B. (1954). On the dissolving power of lecithin and bile salts for cholesterol in human bladder bile. Acta Soc. Med. upsalien, 59, 296-306.

Iwata, T., and Yamasaki, K. (1964). Enzymatic determination and thin-layer chromatography of bile acids in blood. J. Biochem. (Tokyo), 56, 424-431.

King, E. J. (1932). The colorimetric determination of phosphorus. Biochem. J., 26, 292-297.

van der Linden, W. (1971). Bile acid pattern of patients with and without gallstones. Gastroenterology, 60, 1144-1145.

Nakayama, F., and van der Linden, W. (1970). Bile from gallbladder harbouring gallstone: can it indicate stone formation? Acta chir. scan ., 136, 605-610.

Niederhiser, D. H., and Roth, H. P. (1968.) Cholesterol solubilization by solutions of bile salts and bile salts plus lecithin. Proc. Soc. exp. Biol. (N.Y.), 128, 221-225.

Pomare, E. W., and Heaton, K. W. (1972). Increased bacteria degradation of bile salts in cholecystectomized subjects. (Abstr.) Gut, 13, 321-322.

Saunders, D. R., and Wells, M. A. (1969). The cholesterol solubilizing capacity of lecithins in aqueous solutions of bile salt. Biochim. biophys. Acta (Amst.), 176, 828-835.

Skipski, V. P., Peterson, R. F., and Barclay, M. (1964). Quantitative analysis of phospholipids by thin-layer chromatography. Biochem. J., 90, 374-378.

Small, D. M. (1968). Current concepts: gallstones. New Engl. J. Med., 279, 588-593.

Smallwood, R. A., Jablonski, P., McLelland, M., and McK. Watts, J. (1972). The intermittent production of abnormal bile in patients with cholesterol gallstones. (Abstr.). Gastroenterology, 62, 814 .

Tera, H. (1960). Stratification of human gallbladder bile in vivo. Acta chir. scand., Suppl., 256.

Thistle, J. L., and Schoenfield, L. J. (1971). Lithogenic bile among young Indian women. New Engl. J. Med. 284, 177-181.

Vlahcevic, Z. R., Bell, C. C., Jr., and Swell, L. (1970). Significance of the liver in the production of lithogenic bile in man. Gastroenterology, 59, 62-69.

Watson, D. (1960). A simple method for the determination of serum cholesterol. Clin. chim. Acta, 5, 637-643.

Yoshimuta, S. (1960). Factors affecting solubility of cholesterol in bile. Fukuoka Acta med., 51, 570. 